\title{
A hybrid fuzzy MCDM approach to maintenance Quality Function Deployment
}

\author{
Davy George Valavi ${ }^{a^{*}}$ and V.R. Pramod ${ }^{\mathrm{b}}$
}

${ }^{a}$ Research Scholar Department of Mechanical Engineering, Karpagam University, Coimbatore, India ${ }^{b}$ Department of Mechanical Engineering, NSS college of Engineering, Palakkad, india

\section{HRO N I C L E}

\begin{tabular}{l}
\hline Article history: \\
Received March 14, 2014 \\
Accepted August 10,2014 \\
Available online \\
August 142014 \\
\hline Keywords: \\
Fuzzy-Analytic hierarchy \\
process(FAHP) \\
Triangular Fuzzy Number(TFN) \\
Maintenance Quality Function \\
Deployment(MQFD)
\end{tabular}

\begin{abstract}
A B S T R A C T
Maintenance Quality Function Deployment (MQFD) is a model, which enhances the synergic power of Quality Function Deployment (QFD) and Total Productive Maintenance (TPM). One of the crucial and important steps during the implementation of MQFD is the determination of the importance or weightages of the critical factors (CF) and sub factors (SF). The CFs and SFs have to be compared precisely for the successful implementation of MQFD. The crisp pair-wise comparison in the conventional Analytical Hierarchy Process (AHP) may be insufficient to determine the degree of weightage of CFs and SFs where vagueness and uncetainties are associated. In this paper, a modification of AHP based MQFD by incorporating fuzzy operations is proposed, which can improve the accuracy of determination of the weightages. A case study showing the applicability of this method is illustrated in this paper.
\end{abstract}

(c) 2015 Growing Science Ltd. All rights reserved.

\section{Introduction}

Lot of changes have taken place in the business scenario after the second world war.Organisations started adopting various models and better methodologies to excel in the competition. In the mid twentieth century, the field of maintenance quality engineering emerged (Decker 1996). This field developed by adopting new approaches(Chan et al., 2005) and in 1970's a new framework was evolved called Total Productive Maintenance (TPM). The TPM couples the principles of maintenance quality engineering and Total Quality Management (TQM) (Sherwin, 2000). The TPM proved successful in achieving a higher degree of maintenance quality (Mekone et al., 2001, Pramod et al., 2007a). Understanding customers' needs and incorporating them in the product is a necessity to meet the customer's increasing dynamic demand for higher degree of quality and customer satisfaction. Quality Function Deployment (QFD) is a technique adopted in TQM to translate customer's voice into technical language (Kathawala \& Motwani, 1994). QFD is found to be successful in many different industries and applications (Carnevalli \& Miguel 2008). Researchers had realized the need to link TPM with QFD to include customers' voice in maintenance quality improvement plan.

* Corresponding author. Tel: +919845383302

E-mail address: davygeorge@gmail.com (D. G. Valavi) 
Pramod et al. (2006) proposed a model called Maintenance Quality Function Deployment (MQFD) to have a synergic gain in maintenance quality by linking TPM with QFD. This model has been validated in different practical scenarios (Pramod et al., 2006, 2007b, 2008). MQFD is modified by including AHP for calculating the weightages of the critical factors and subfactors (Pramod et al., 2007b). The AHP evaluates the critical factors using crisp pair-wise comparisons (Saaty, 1994, 2008).

Evaluation of the relative importance or weightages of customer needs/critical factors is a critical step in MQFD process. Most of the decision making in the real world takes place in situations where the vagueness are associated with data and the sequence of possible actions are not accurately known. Triantaphyllou and Lin (1996) suggested that it is better to use fuzzy data to represent such situations in decision making problems where vagueness and uncertainity are associated. Due to the imprecision existing in judgement of the decison makers, the crisp pair-wise comparison in the conventional AHP may be insufficient to assess the degree of importance of customer requirements (Kwong \& Bai, 2002; Triantaphyllou \& Lin, 1996). Yang and Zhang(2010) stated that Fuzzy-AHP (FAHP) is a powerful and flexible multi-criteria decision making (MCDM) tool for dealing with complex problems where both qualitative and quantitative aspects are to be evaluated and when the experts judgements are vague. Accurate prioritisation of customer needs and technical requiremnents will lead to a better implemntation of MQFD.

Based on the above considerations, a fuzzy based AHP-MQFD is proposed in this paper. This model aims to evaluate the importance ratings (weightages) of CFs and SFs in MQFD. This paper is arranged as follows. MQFD is illustrated in section 2, Fuzzy -AHP MQFD is explained in section 3. Section 4 explains case study, Results and conclusions are in the subsequent sections.

\section{Maintenance Quality Function Deployment}

The major features of MQFD are described in this section. The MQFD framework is shown in Figure 1. The customers' voice is gathered which are then used by the QFD team to develop the House of Qualtiy(HoQ) (Chein \& Su, 2003). HoQ is a tool to translate customers voice into technical requirements, These requirements are submitted to the management for making strategic decisions. Researchers has emphasized that the strategic approach is essential for the success of QFD and TPM projects. (Lu \& Kuei, 1998, Hunt \& Xavier 2003).

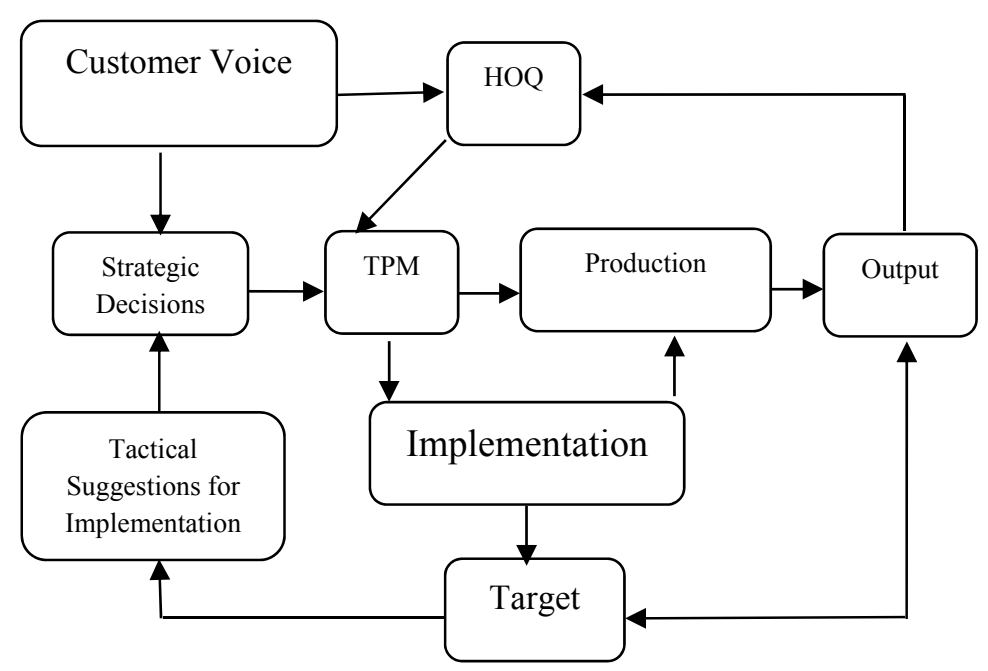

Fig. 1. MQFD model 
The technical requirements concerning the improvement of maintenance quality are processed through the eight pillars of TPM to develop TPM methodologies. These methodologies are then applied in the production system. Their implementations is to be focussed on increasing the values of the maintenance quality parameters such as Overall Equipment Efficiency (OEE), Mean Time Between Failures (MRBF), Mean Time To Repair (MTTR), Performance Quality (PQ),Availability and Mean Down Time (MDT).

The outputs are used to compare with the set targets and to develop HoQ for the next cycle. The result of the implementation of MQFD will be the improvement in maintenance quality, enhancement in profit etc. The implementation of MQFD model is a continuous improvement process. A unique feature of the MQFD model is that it does not necessitate extensive changes in the existing continuous improvement processes like TQM and TQM which may be practiced in the company. Thus, MQFD model enables the link between QFD and TPM. For further illustrations about MQFD (See for instance, Pramod et al., 2006, 2007b, 2008).

\section{Fuzzy-AHP MQFD Model}

The Analytic Hierarchy Process (AHP) is a systematic technique for organizing and analyzing complex decisions. It was developed by Thomas L Saaty in the 1970s and has been studied and modified by the researchers. There are three steps of AHP methodology (Saaty, 1994, 2008), structuring the hierarchy, performing the comparative pair-wise judgement and synthesising results. Fuzzy set theory can be used in situatations where uncertainty and ambiguity are associated with the mapping of decision maker's judgement to crisp numbers (Mechefske \& Wang, 2001 Yang; Zhang, 2010). This necessitated the development of Fuzzy-AHP (FAHP) model. FAHP has been applied in different areas like Information System (Thalia et al., 2012), Maintenance Management (Chun et al., 2012), Risk Management (Ni et al., 2010), Supply Chain Management (Yang \& Zhang, 2010, Wang \& Luan 2012), etc.

In the conventional AHP eigen vectors are used to calculate the final weightages. However, Lootsma (1988) suggested that normalized column and row weights are equivalent to normalized eigen vectors. Verma et al. (2007) proposed that average of two normalized weights can be considered as final weightages. Gaonkar et al. (2008) has shown that this method can be applied for solving maintenance strategy selection problem. This approach is used in this work to enhance the AHPMQFD model to fuzzy based AHP- MQFD model. In AHP, a group of experts would fill the pairwise comparison matrix. Alternatively, experts would give the importance for each criteria in a scale, (usually 1-9) and then the average values are converted into equivalent Saaty's score using equation (7). The conversion of values into scores of Saaty's scale is a vital step in AHP (Karapetrovia \& Rosenbloom 1999). In this model, scores of saaty"s scale are converted into triangular fuzzy numbers. Fig. 2 illustrates the stepes involved in this proposed model to determine the weightages of CFs and SFs.

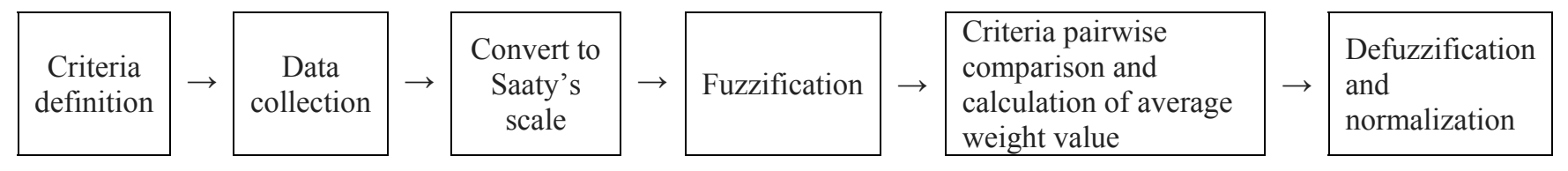

Fig. 2. Steps in the proposed model to determine the weightages

A fuzzy number $M$ on $R \varepsilon[-\alpha,+\alpha]$ is defined to be a triangular fuzzy number if its membership function $\mu_{m} R \rightarrow[0,1]$ is equal to 


$$
\begin{array}{ll}
\mu_{m}(x)=\left\{\frac{1}{m-l}(x)-\frac{l}{m-l}\right. & x \varepsilon[l, m] \\
\mu_{m}(x)=\left\{\frac{1}{m-u}(x)-\frac{l}{m-u}\right. & x \varepsilon[m, u]
\end{array}
$$

Table 1

Saaty's Scales $(1-9)$ expressed as Triangular Fuzzy Number(TFN)

\begin{tabular}{llc}
\hline Scale & Defination & Membership values \\
\hline 1 & Equally Important & $(1,1,2)$ \\
3 & Moderate more Important & $(2,3,4)$ \\
5 & Strongly more Important & $(4,5,6)$ \\
7 & Very strongly more important & $(6,7,8)$ \\
9 & Exceedingly more important & $(8,9,9)$ \\
\hline
\end{tabular}

where $l \leq m \leq u, l$ and $\mathrm{u}$ stand for lower and upper values of the support of the fuzzy number $M$, respectively and $\mathrm{m}$ for the modal value (Triantaphyllou \& Lin, 1996; Ross, 2010). A fuzzy number, as expressed by Eq. (1) will be denoted by $\left(x_{l}, x_{m}, x_{u}\right)$. Table 1 shows the Saaty's Scales expressed as Triangular Fuzzy Number(TFN). The fuzzy numbers are used to construct pair-wise comparison matrix as follows:

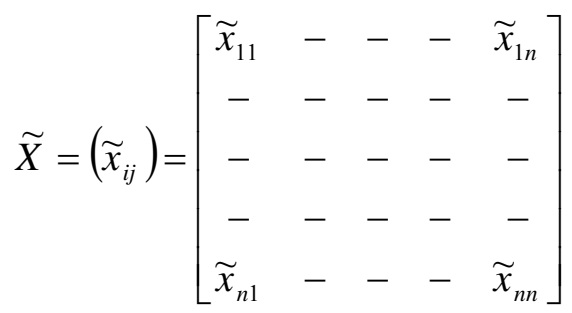

where $n$ is the number of criteria, $\widetilde{x}_{i j}=\left(\widetilde{x}_{j i}\right)^{-1}$ and $\quad \widetilde{x}_{i j}=1$ if $i=j$. The basic operations on fuzzy triangular number are explained in (Dehghanian et al., 2012, Ross, 2010; Triantaphyllou \& Lin, 1996) are given below.

$$
\begin{aligned}
& \widetilde{x}_{1} \oplus \tilde{x}_{2}=\left(x_{1 l}+x_{2 l}, x_{1 m}+x_{2 m}, x_{1 u}+x_{2 u}\right) \\
& \tilde{x}_{1} \otimes \tilde{x}_{2}=\left(x_{1 l} \times x_{2 l}, x_{1 m} \times x_{2 m}, x_{1 u} \times x_{2 u}\right) \\
& -\widetilde{x}_{1}=\left(-x_{1 l},-x_{1 m},-x_{1 u}\right) \\
& \frac{1}{\widetilde{x}_{1}} \cong\left(\frac{1}{x_{1 u}}, \frac{1}{x_{1 m}}, \frac{1}{x_{1 l}}\right)
\end{aligned}
$$

where $\cong$ denotes approximation, $\widetilde{x}_{1}=\left(x_{1 l}, x_{1 m}, x_{1 u}\right)$ and $\widetilde{x}_{2}=\left(x_{2 l}, x_{2 m}, x_{2 u}\right)$ respresent two fuzzy triangular numbers with lower, modal and upper values.

$$
Y=1+\left[\left(x-x_{\text {min }}\right) \times \frac{8}{\left(x_{\text {max }}-x_{\text {min }}\right)}\right]
$$

where $\mathrm{Y}=$ equivalent score in Saaty's 1-9 scale, $x=$ average values computed against each criticalfactor/sub factor, 
$x_{\min }=$ minimum average value in each group critical factor/subfactor,

$x_{\max }=$ maximum average factor in each group criticalfactor/subfactor.

These scores are used to construct the pairwise comparison matrix in conventional AHP. However, in this fuzzy model these scores are converted into triangular fuzzy numbers. After the fuzzification of data, a fuzzy pairwise comparision matrix is constructed using the Eq. (2). $\tilde{x}_{\mathrm{ij}}$ dentoes the fuzzy values assigned to the relative importance of criteria $C_{i}$ to $C_{j}$. These values are obtained by calculating the ratio of fuzzy number associated with $C_{i}$ to the fuzzy number associated with $C_{j}$.

The average weightages of all criteria are calculated using the Eq. (8) to Eq. (13).

Row Sum, $r \widetilde{s}_{i}=\sum_{j=1}^{n} \widetilde{x}_{i j} \quad \forall \quad i=1,2,3 \ldots \ldots \ldots \ldots, n$

Column Sum, $c \widetilde{s}_{j}=\sum_{i=1}^{n} \widetilde{x}_{i j} \quad \forall \quad j=1,2,3 \ldots \ldots ., n$

Cumulative Row Sum, $c r \widetilde{s}=\sum_{i=1}^{n} r \widetilde{s}_{i}$

Normalized Row Vector $\widetilde{n}_{i}=r \widetilde{s}_{i} \div c r \widetilde{s}$

Normalized Column Vector, $i \widetilde{n}_{i}=\left(c s_{i}\right)^{-1}$

Average weightage of criteria $i, \quad \widetilde{w}_{i}=\left(\frac{\widetilde{n}_{i} \oplus i \widetilde{n}_{i}}{2}\right)$

Defuzzification is the process of converting a fuzzy number to a crisp number. In this work, the center of gravity method (Ross, 2010), as given by Eq. (14), is adopted for defuzzification.

$w_{i}^{d}=\left(\frac{\left(w_{u}-w_{l}\right)+\left(w_{m}-w_{l}\right)}{3}\right)+w_{l}, \forall i=1,2, \ldots, n$

Normalization, $\quad \bar{w}_{i}=\frac{w_{i}^{d}}{\sum_{i=1}^{n} w_{i}^{d}}, \forall \quad i=1,2,3 \ldots ., n$

\section{Case Study}

Pramod,V.R et.al (2007b) had conducted a study in a public sector automobile service station at coimbatore,India, to examine the practicality of AHP based MQFD. The service station is required to cater to the maintennance requirements of government owned vehicles. This study is based on that work and focuses on the determination of importance ratings(weightages) and ranking of CFs and SFs. In that study the MQFD was decomposed into five components namely, house of quality, decision systems, TPM, maintenance parameters and quality parameters. They used a 9 point scale to collect the data from the customers(competent personnel in the service station). The average score obtained was then converted into Saaty's scale. The components, CFs, SFs,average score and Saaty's score in that study is shown Table2. In this work the Saaty's score are converted to Triangular Fuzzy Number (TFN). The corresponding TFNs assigned are given in the last coloumn of Table 2. The TFN is assigned in such a manner that the lower value is not less than 1 and upper value is not greater than 9. The Saaty's score is taken as the corresponding modal value. A sample calculation for the component HoQ is shown in Table 3 and Table 4. 


\section{Table 2}

Score in Saaty's Scale and Corresponding TFN

\begin{tabular}{|c|c|c|c|c|c|}
\hline Component & Critical Factors & Sub-factors & Avg. Value & Score in Saaty's Scale & TFN \\
\hline \multirow{21}{*}{ HoQ } & Customer (C1) & & 7.9 & 9.0 & 8.09 .09 .0 \\
\hline & & Freequency of vehicle breakdown(S1) & 6.4 & 4.4 & 3.44 .45 .4 \\
\hline & & Cultural Background(S2) & 5.3 & 1.0 & 1.01 .02 .0 \\
\hline & & Duration of maintenance (S3) & 6.0 & 3.2 & 2.23 .24 .2 \\
\hline & & Emergency necessity(S4) & 6.5 & 4.7 & 3.74 .75 .7 \\
\hline & & Quality of spare parts(S5) & 7.9 & 9.0 & 8.09 .09 .0 \\
\hline & & Cost of spare parts(S6) & 6.2 & 3.8 & 2.83 .84 .8 \\
\hline & Technology (C2) & & 7.3 & 5.6 & 4.65 .66 .6 \\
\hline & & Infrastructure (S7) & 7.7 & 6.8 & 5.86 .87 .8 \\
\hline & & Skill of the personal(S8) & 8.2 & 9.0 & 8.09 .09 .0 \\
\hline & & Employer employee relationship(S9) & 7.3 & 5.0 & $4.05 .0 \quad 6.0$ \\
\hline & & Organisational climate(S10) & 6.4 & 1.0 & $\begin{array}{lll}1.0 & 1.0 & 2.0\end{array}$ \\
\hline & & Maintenance methods(S11) & 6.8 & 2.8 & 1.82 .83 .8 \\
\hline & Competitors (C3) & & 6.5 & 1.0 & 1.01 .02 .0 \\
\hline & & Financial power(S12) & 8.0 & 9.0 & 8.09 .09 .0 \\
\hline & & Performance of competitors(S13) & 7.4 & 7.8 & $6.87 .8 \quad 8.8$ \\
\hline & & Customer relationship(S14) & 7.7 & 8.4 & 7.48 .49 .0 \\
\hline & & Strategies of competitors(S15) & 5.7 & 4.5 & $3.54 .5 \quad 6.5$ \\
\hline & & Change management scheme(S16) & 5.9 & 1.0 & $\begin{array}{lll}1.0 & 1.0 & 2.0\end{array}$ \\
\hline & & Quality parameters(S17) & 7.4 & 7.8 & $6.87 .8 \quad 8.8$ \\
\hline & & New Technology(S18 & 7.4 & 7.8 & $6.87 .8 \quad 8.8$ \\
\hline & Personnel Factor (C & & 8.1 & 9.0 & 8.09 .09 .0 \\
\hline & & Authority of personal(S19) & 7.0 & 1.0 & 1.01 .02 .0 \\
\hline Decision system & & Responsibility of Personal (S20) & 8.3 & 9.0 & 8.09 .09 .0 \\
\hline & & Initiatives of personal(S21) & 7.4 & 3.5 & $2.5 \quad 3.5 \quad 4.5$ \\
\hline & & Motivation of personal(S22) & 8.0 & 7.2 & 6.27 .28 .2 \\
\hline & Value of Decisions & & 6.3 & 1.0 & 1.01 .02 .0 \\
\hline & Reliability of Decis & ns (C6) & 7.0 & 4.1 & 3.14 .15 .1 \\
\hline & Autonomous Maint & ance (C7) & 7.7 & 9.0 & $\begin{array}{lll}8.09 .09 .0 \\
\end{array}$ \\
\hline & & Atitude of workers(S23) & 7.1 & 6.6 & 5.66 .67 .6 \\
\hline & & Atitude of management(S24) & 7.1 & 6.6 & 5.66 .67 .6 \\
\hline & & Motivation schemes(S25) & 5.5 & 1.0 & 1.01 .02 .0 \\
\hline & & Incentive of salary(S26) & 7.8 & 9.0 & 8.09 .09 .0 \\
\hline & & Financial benefits(S27) & 7.2 & 6.9 & 5.96 .97 .9 \\
\hline & & Lubrication management(S28) & 6.3 & 3.8 & 2.83 .84 .8 \\
\hline & & Daily maintenance of data(S29) & 6.9 & 5.9 & 4.95 .96 .9 \\
\hline TPM & Individual Improve & ent (C8) & 7.0 & 6.6 & 5.66 .67 .6 \\
\hline & & Reputation of individual (S30) & 7.3 & 3.9 & 2.93 .94 .9 \\
\hline & & Kaizen's principle(S31) & 8.5 & 9.0 & 8.09 .09 .0 \\
\hline & & Employee's suggestion scheme(S32) & 6.8 & 1.8 & $\begin{array}{lll}1.0 & 1.8 & 2.8\end{array}$ \\
\hline & & Employee involvement scheme(S33) & 7.0 & 2.7 & 1.72 .73 .7 \\
\hline & & Daily maintenance of machinery(S34) & 6.6 & 1.0 & $\begin{array}{lll}1.0 & 1.0 & 2.0\end{array}$ \\
\hline & & Interpersonal relationship(S35) & 7.3 & 3.9 & 2.93 .94 .9 \\
\hline & & Employee's wish(S36) & 7.0 & 2.7 & 1.72 .73 .7 \\
\hline & Planned Maintenan & (C9) & 7.6 & 8.7 & 7.78 .79 .0 \\
\hline & & Schedule of maintenance(S37) & 7.5 & 7.9 & 6.97 .98 .9 \\
\hline & & Freequency of breakdown(S38) & 7.7 & 9.0 & 8.09 .09 .0 \\
\hline & & Idleness of machine(S39) & 6.3 & 1.0 & 1.01 .02 .0 \\
\hline & & Repetition of same problem(S40) & 6.8 & 3.9 & 2.93 .94 .9 \\
\hline & Quality Maintenan & (C10) & 7.5 & 8.3 & $7.38 .3 \quad 8.3$ \\
\hline & & $\mathrm{TQM}$ tools(S41) & 6.7 & 7.3 & 6.37 .38 .3 \\
\hline & & Sampling(S42) & 2.4 & 1.0 & $\begin{array}{lll}1.0 & 1.0 & 2.0\end{array}$ \\
\hline & & Data management(S43) & 7.9 & 9.0 & 8.09 .09 .0 \\
\hline & Education And Tra & ing $(\mathrm{C} 11)$ & 7.5 & 8.3 & 7.38 .39 .0 \\
\hline & & Feasibility for higher studies(S44) & 6.3 & 1.0 & $\begin{array}{lll}1.0 & 1.0 & 2.0\end{array}$ \\
\hline & & Training facility(S45) & 7.4 & 6.5 & $5.5 \quad 6.5 \quad 7.5$ \\
\hline & & Employee's own interest(S46) & 7.4 & 6.5 & $5.5 \quad 6.57 .5$ \\
\hline & & Motivation for training(S47) & 7.1 & 5.0 & $4.05 .0 \quad 6.0$ \\
\hline & & Rewards for better performance(S48) & 7.9 & 9.0 & 8.09 .09 .0 \\
\hline & Development mana & ment (C12) & 6.6 & 5.1 & $4.15 .1 \quad 6.1$ \\
\hline & & Target setting(S49) & 7.5 & 9.0 & 8.09 .09 .0 \\
\hline & & Job scheduling(S51) & 6.7 & 7.6 & 6.67 .68 .6 \\
\hline & & Production planning(S51) & 2.9 & 1.0 & $\begin{array}{lll}1.0 & 1.0 & 2.0\end{array}$ \\
\hline & & Maintenance schedule(S52) & 7.3 & 8.7 & 7.78 .79 .0 \\
\hline & Office TPM (C13) & & 5.4 & 1.0 & $\begin{array}{lll}1.0 & 1.0 & 2.0\end{array}$ \\
\hline & & New Technology(S53) & 8.1 & 9.0 & 8.09 .09 .0 \\
\hline & & Training(S54) & 7.5 & 7.3 & $6.37 .3 \quad 8.3$ \\
\hline & & Motivation(S55) & 6.8 & 5.4 & $4.45 .4 \quad 6.4$ \\
\hline & & Proximity of customer(S56) & 5.2 & 1.0 & $\begin{array}{lll}1.0 & 1.0 & 2.0\end{array}$ \\
\hline & & Proximity of supplier(S57) & 5.8 & 2.7 & 1.72 .73 .7 \\
\hline & & Data processing speed(S58) & 7.7 & 7.9 & 6.97 .98 .9 \\
\hline & Safety Health And & 1vironment (C14) & 7.5 & 8.3 & 7.38 .39 .0 \\
\hline & & Hospital(59) & 5.0 & 1.0 & 1.01 .02 .0 \\
\hline & & Gymnasium(S60) & 5.4 & 2.1 & 1.12 .13 .1 \\
\hline & & Pollution(S61) & 7.3 & 7.6 & 6.67 .68 .6 \\
\hline & & Safety rules(S62) & 7.8 & 9.0 & 8.09 .09 .0 \\
\hline & & Green belt concepts(S63) & 6.7 & 5.9 & 4.95 .96 .9 \\
\hline & & Safety training(S64) & 7.1 & 7.0 & 6.07 .08 .0 \\
\hline & & Display for safety(S65) & 7.0 & 6.7 & 5.76 .77 .7 \\
\hline & & Periodic medical check up(S66) & 6.5 & 5.3 & $4.35 .3 \quad 6.3$ \\
\hline & Overall equipment & fectiveness (C15) & 7.3 & 6.7 & 5.76 .77 .7 \\
\hline & Mean time between & ailure (C16) & 5.6 & 1.0 & 1.01 .02 .0 \\
\hline Mainten-ance & Mean time to repair & (C17) & 6.0 & 2.3 & $\begin{array}{lll}1.3 & 2.3 & 3.3\end{array}$ \\
\hline Parmeters & Performance efficie & $\operatorname{cies}(\mathrm{C} 18)$ & 8.0 & 9.0 & $\begin{array}{lll}8.0 & 9.0 & 9.0\end{array}$ \\
\hline & Mean down time (C & & 7.0 & 5.7 & $4.75 .7 \quad 6.7$ \\
\hline & Availability (C20) & & 7.1 & 6.0 & $\begin{array}{llll}5.0 & 6.0 & 7.0 \\
\end{array}$ \\
\hline & Improved Maintena & ce (C21) & 8.0 & 9.0 & $\begin{array}{lll}8.0 & 9.0 & 9.0\end{array}$ \\
\hline Quality paramet-ers & Increased Profit (C & & 5.3 & 1.0 & $\begin{array}{lll}1.0 & 1.0 & 2.0\end{array}$ \\
\hline & Upgraded core com & etence (C23) & 5.5 & 4.7 & 3.74 .75 .7 \\
\hline & Enhanced good wil & (C24) & 8.0 & 9.0 & $\begin{array}{lll}8.0 & 9.0 & 9.0\end{array}$ \\
\hline
\end{tabular}

The value of cumulative row sum $\widetilde{\boldsymbol{c r} s}=\left(\begin{array}{l}11.2820 .1222 .07\end{array}\right)$ 
Table 3

Fuzzy Pair-Wise Comparison Matrix of the Component HoQ

\begin{tabular}{ccccc}
\hline Criteria & $\mathrm{C} 1$ & $\mathrm{C} 2$ & $\mathrm{C} 3$ & $\widetilde{r S}$ \\
\hline $\mathrm{C} 1$ & 111 & 1.211 .611 .96 & 4.09 .09 .0 & 6.2111 .6111 .96 \\
$\mathrm{C} 2$ & .51 .62 .83 & 111 & 2.35 .66 .6 & 3.817 .228 .43 \\
$\mathrm{C} 3$ & .11 .11 .25 & .15 .18 .43 & 111 & 1.261 .291 .68 \\
$\widetilde{C S}$ & 1.621 .732 .08 & 2.362 .793 .39 & 7.315 .616 .6 & 11.2820 .1222 .07 \\
\hline
\end{tabular}

Table 4

Final Weightages of the Critical Factors of HoQ

\begin{tabular}{cccc}
\hline Criteria & $\mathrm{C} 1$ & $\mathrm{C} 2$ & $\mathrm{C} 3$ \\
\hline$\widetilde{r S}_{\mathrm{i}}$ & 6.2111 .6111 .96 & 3.817 .228 .43 & 1.261 .291 .68 \\
$\widetilde{\widetilde{C S}}_{\mathrm{i}}$ & 1.621 .732 .08 & 2.362 .793 .39 & 7.315 .616 .6 \\
$\widetilde{n}_{\mathrm{i}}$ & .28 .581 .06 & .17 .36 .75 & .06 .06 .15 \\
$\widetilde{\imath}_{\mathrm{i}}$ & .48 .58 .62 & .29 .36 .43 & .06 .06 .14 \\
$\widetilde{W}_{\mathrm{i}}^{d}$ & .38 .58 .84 & .23 .36 .59 & .06 .06 .15 \\
$w_{i}^{d}$ & .60 & .39 & .09 \\
$\bar{w}_{\mathrm{i}}$ & .56 & .36 & .08 \\
\hline
\end{tabular}

These calculations are carried out for the other components and critical facors. The results are tabulated in the Table 5 and Table 6 . The global weightages of subfactors are calculated by multiplying the local weightages of the subfactors and the corresponding local weightages of the critical factors.

\section{Table 5}

Local Weightages of Critical Factors of MQFD model

\begin{tabular}{lccccc}
\hline $\begin{array}{l}\text { Critical } \\
\text { Factors }\end{array}$ & $\begin{array}{c}\text { Local sensitivity } \\
\text { AHP - MQFD }\end{array}$ & $\begin{array}{c}\text { Local sensitivity } \\
\text { FAHP -MQFD }\end{array}$ & \% Change & $\begin{array}{c}\text { Rank. } \\
\text { AHP - MQFD }\end{array}$ & $\begin{array}{c}\text { Rank } \\
\text { FAHP -MQFD }\end{array}$ \\
\hline C1 & 0.62 & .56 & 9.7 & 1 & 2 \\
C2 & 0.32 & .36 & 12.5 & 5 & 5 \\
C3 & 0.06 & .08 & 33.3 & 20 & 20 \\
C4 & 0.60 & .61 & 1.7 & 2 & 1 \\
C5 & 0.08 & .09 & 12.5 & 19 & 19 \\
C6 & 0.32 & .30 & 6.3 & 6 & 12 \\
C7 & 0.15 & .16 & 6.7 & 12 & 17 \\
C8 & 0.14 & .12 & 14.3 & 17 & 13 \\
C9 & 0.15 & .15 & 0.0 & 13 & 14 \\
C10 & 0.15 & .15 & 0.0 & 14 & 15 \\
C11 & 0.15 & .15 & 0.0 & 15 & 18 \\
C12 & 0.09 & .10 & 11.1 & 18 & 24 \\
C13 & 0.02 & .02 & 0.0 & 24 & 8 \\
C14 & 0.15 & .15 & 0.0 & 16 & 23 \\
C15 & 0.23 & .22 & 4.3 & 8 & 21 \\
C16 & 0.03 & .04 & 33.3 & 23 & 7 \\
C17 & 0.06 & .08 & 33.3 & 21 & 11 \\
C18 & 0.28 & .28 & 0.0 & 7 & 10 \\
C19 & 0.21 & .19 & 9.5 & 9 & 3 \\
C20 & 0.19 & .20 & 5.3 & 11 & 22 \\
C21 & 0.38 & .37 & 2.6 & 3 & 9 \\
C22 & 0.04 & .06 & 50.0 & 22 & 4 \\
C23 & 0.20 & .21 & 5.0 & 10 & 4 \\
C24 & 0.38 & .37 & 2.6 & 4 & \\
\hline & & & & & \\
\hline
\end{tabular}




\section{Table 6}

Local and Global Weightages of Subfactors of MQFD model

\begin{tabular}{|c|c|c|c|c|c|c|}
\hline Sub factors & Global weightage & Local weightage & Global weightage & $\%$ Diff. & Rank & Rank \\
\hline S1 & .097 & .17 & .095 & 2.1 & 6 & 8 \\
\hline S2 & .024 & .05 & .028 & 16.7 & 31 & 26 \\
\hline S3 & .077 & .12 & .067 & 13.0 & 11 & 12 \\
\hline S4 & .113 & .18 & .101 & 10.6 & 4 & 6 \\
\hline S5 & .208 & .33 & .185 & 11.1 & 3 & 3 \\
\hline S6 & .097 & .15 & .084 & 13.4 & 7 & 9 \\
\hline S7 & .082 & .28 & .101 & 23.2 & 10 & 7 \\
\hline S8 & .106 & .35 & .126 & 18.9 & 5 & 4 \\
\hline S9 & .083 & .21 & .076 & 8.4 & 9 & 10 \\
\hline $\mathrm{S} 10$ & .012 & .05 & .018 & 50.0 & 45 & 40 \\
\hline S1 1 & .036 & .11 & .040 & 11.1 & 18 & 17 \\
\hline $\mathrm{S} 12$ & .012 & .19 & .015 & 25.0 & 46 & 43 \\
\hline $\mathrm{S} 13$ & .011 & .17 & .014 & 27.3 & 48 & 44 \\
\hline S14 & .011 & .18 & .014 & 27.3 & 49 & 45 \\
\hline S15 & .007 & .10 & .008 & 14.3 & 54 & 53 \\
\hline S16 & .002 & .03 & .002 & 0.0 & 63 & 64 \\
\hline S17 & .011 & .17 & .014 & 27.3 & 50 & 46 \\
\hline $\mathrm{S} 18$ & .012 & .17 & .014 & 16.7 & 47 & 47 \\
\hline S19 & .031 & .06 & .037 & 19.4 & 21 & 19 \\
\hline S20 & .258 & .42 & .256 & 0.8 & 1 & 1 \\
\hline S21 & .095 & .17 & .104 & 9.5 & 8 & 5 \\
\hline S22 & .219 & .35 & .214 & 2.3 & 2 & 2 \\
\hline S23 & .026 & .17 & .027 & 3.8 & 27 & 28 \\
\hline S24 & .026 & .17 & .027 & 3.8 & 28 & 29 \\
\hline S25 & .004 & .03 & .005 & 25.0 & 59 & 57 \\
\hline S26 & .031 & .22 & .035 & 12.9 & 22 & 20 \\
\hline S27 & .026 & .17 & .027 & 3.8 & 29 & 30 \\
\hline S28 & .013 & .10 & .016 & 23.1 & 44 & 42 \\
\hline S29 & .026 & .15 & .024 & 7.7 & 30 & 34 \\
\hline $\mathrm{S} 30$ & .020 & .16 & .019 & 5.0 & 38 & 38 \\
\hline S31 & .047 & .33 & .040 & 14.9 & 16 & 18 \\
\hline S32 & .010 & .08 & .010 & 0.0 & 51 & 51 \\
\hline S33 & .018 & .11 & .013 & 27.8 & 41 & 48 \\
\hline S34 & .005 & .05 & .006 & 20.0 & 57 & 56 \\
\hline S35 & .019 & .16 & .019 & 0.0 & 40 & 39 \\
\hline S36 & .018 & .11 & .013 & 27.8 & 42 & 49 \\
\hline S37 & .055 & .36 & .054 & 1.8 & 15 & 15 \\
\hline S38 & .062 & .40 & .060 & 3.2 & 14 & 14 \\
\hline S39 & .016 & .06 & .009 & 43.8 & 43 & 52 \\
\hline S40 & .020 & .18 & .027 & 35.0 & 39 & 31 \\
\hline S41 & .067 & .42 & .063 & 6.0 & 13 & 13 \\
\hline S42 & .009 & .07 & .011 & 22.2 & 52 & 50 \\
\hline S43 & .074 & .50 & .076 & 2.7 & 12 & 11 \\
\hline S44 & .009 & .05 & .008 & 11.1 & 53 & 54 \\
\hline S45 & .033 & .23 & .035 & 6.1 & 19 & 21 \\
\hline S46 & .033 & .23 & .035 & 6.1 & 20 & 22 \\
\hline S47 & .031 & .18 & .027 & 12.9 & 23 & 32 \\
\hline S48 & .044 & .31 & .045 & 2.3 & 17 & 16 \\
\hline S49 & .029 & .33 & .033 & 13.8 & 25 & 23 \\
\hline S50 & .029 & .29 & .030 & 3.4 & 26 & 25 \\
\hline S51 & .007 & .05 & .005 & 28.6 & 55 & 58 \\
\hline S52 & .021 & .32 & .032 & 52.4 & 35 & 24 \\
\hline S53 & .005 & .26 & .005 & 0.0 & 58 & 29 \\
\hline S54 & .004 & .22 & .004 & 0.0 & 60 & 62 \\
\hline S55 & .002 & .16 & .003 & 50.0 & 64 & 63 \\
\hline S56 & .001 & .04 & .001 & 0.0 & 66 & 66 \\
\hline S57 & .002 & .08 & .002 & 0.0 & 65 & 65 \\
\hline S58 & .004 & .24 & .005 & 25.0 & 61 & 60 \\
\hline S59 & .003 & .03 & .005 & 66.7 & 62 & 61 \\
\hline S60 & .006 & .05 & .008 & 33.3 & 56 & 55 \\
\hline S61 & .024 & .17 & .026 & 8.3 & 32 & 33 \\
\hline S62 & .030 & .19 & .028 & 6.7 & 24 & 27 \\
\hline S63 & .021 & .13 & .020 & 4.8 & 36 & 37 \\
\hline S64 & .024 & .16 & .022 & 8.3 & 33 & 36 \\
\hline S65 & .024 & .15 & .023 & 4.2 & 34 & 35 \\
\hline S66 & .021 & .12 & .018 & 14.3 & 37 & 41 \\
\hline
\end{tabular}




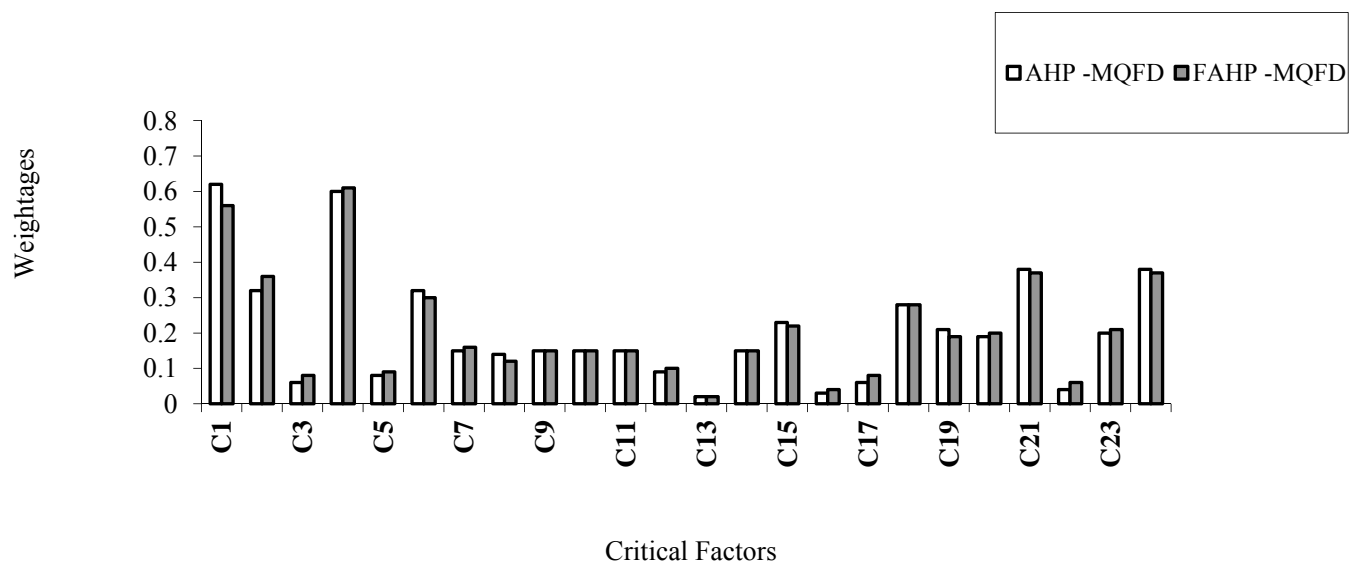

Fig. 3. Difference in local weightages of critical factors

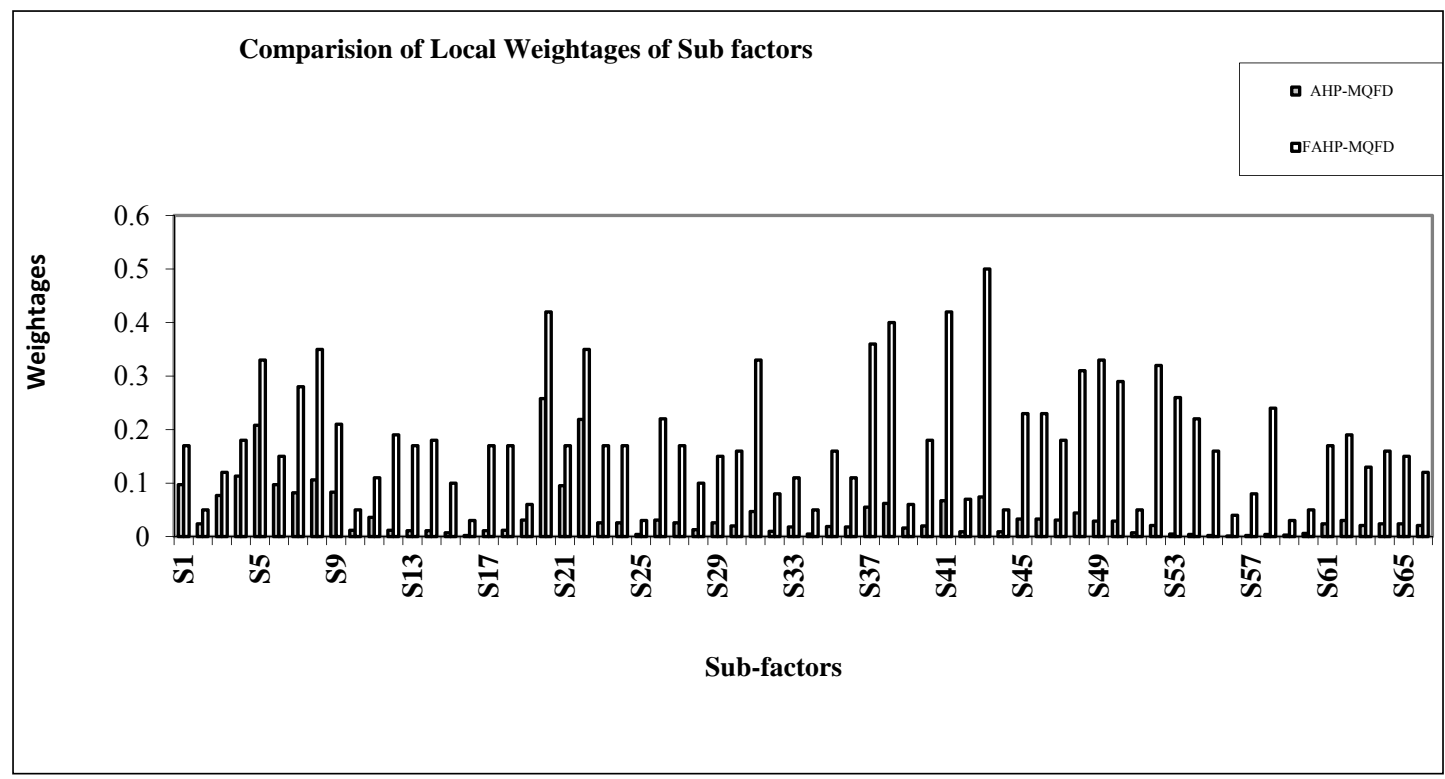

Fig. 4. Difference in global weightages of subfactors

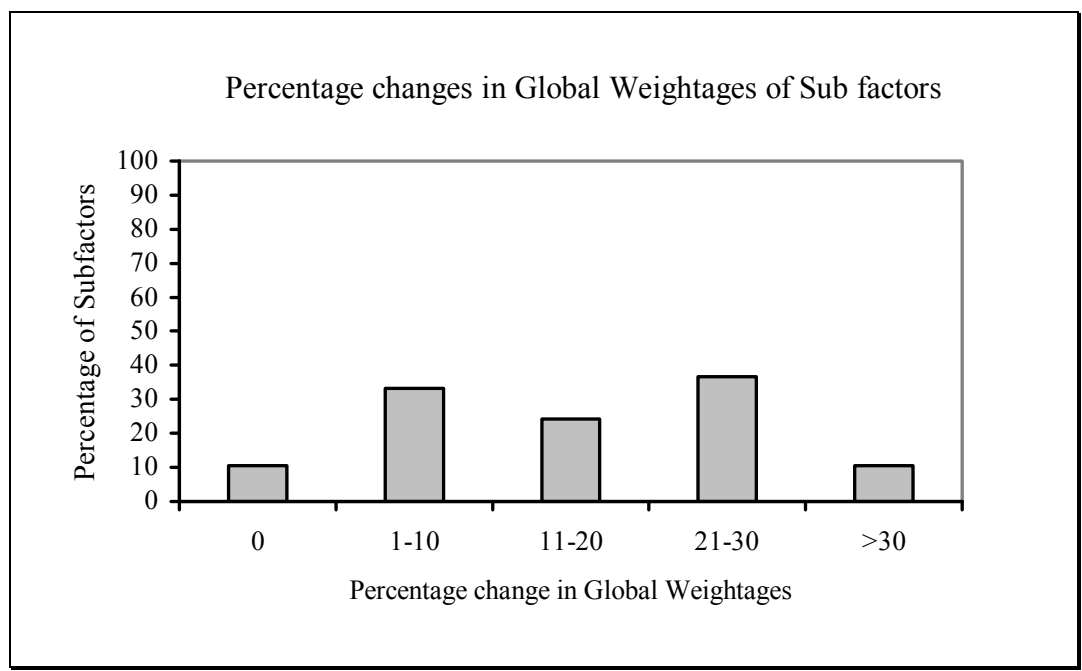

Fig. 5. Percentage difference in global weightages of subfactors 


\section{Results}

The results obtained by the Fuzzy-AHP MQFD model are tabulated in Table 5 and Table 6. For comparison purpose the corresponding values obtained using the crisp AHP method (Pramod et al., 2007) are also shown. The result showed that the critical factor (C4), the personal factor, has the highest ranking when fuzzy AHP method is used. Whereas critical factor customer $(C 1)$ had the highest ranking in the crisp AHP method. The percentage difference in local weightages of the CFs are shown in Fig. 3. The average difference of weightages of CFs between the two methods is about $11 \%$ per factor. There is a marked difference in the rank orders of the SFs between the two models as shown in Table 6. The percentage difference in global weightages of the SFs are shown in Fig. 4 and Fig. 5. The average difference in the global weightages of SFs between the two methods is about $15 \%$ per SF. The results showed that there is significant difference in the values of weightages and rank orders obtained by the two methods namely, AHP - MQFD and Fuzzy AHP - MQFD methods.

\section{Conclusions}

This research work proposes a fuzzy AHP MQFD methodology by integrating fuzzy arithmetic operations with crisp AHP MQFD.The model used in this paper provides both ranking and weighting informations by fuzzy AHP-MQFD. Working with fuzzy numbers and performing fuzzy arithmetic provide great flexibility in decision making. This model successfully addresses the main drawback of crisp AHP-MQFD model in representing and processing the vague customer needs to determine the weightages od CFs and SFs. Effective and efficient prioritisation of CFs and SFs can facilitate better implementaion of MQFD. Hence it is concluded that the integration of fuzzy AHP to MQFD is a good proposition to achieve the successful results of MQFD implementation.

\section{Acknowledgement}

The authors would like to thank the annonymous referees for constructive comments on earlier version of this paper.

\section{References}

Carnevalli, J. A., \& Miguel, P. C. (2008). Review, analysis and classification of the literature on QFD-Types of research, difficulties and benefits. International Journal of Production Economics, 114(2), 737-754.

Chan, F. T. S., Lau, H. C. W., Ip, R. W. L., Chan, H. K., \& Kong, S. (2005). Implementation of total productive maintenance: a case study. International Journal of Production Economics, 95(1), 7194.

Chien, T. K., \& Su, C. T. (2003). Using the QFD concept to resolve customer satisfaction strategy decisions. International Journal of Quality \& Reliability Management, 20(3), 345-359.

Chun, Y., Qi-dong, Y., Li-bin, G., \& Bing-bin, C. (2012, June). Research on the maintenance management performance appraisal of oil equipments based on fuzzy AHP method. In Robotics and Applications (ISRA), 2012 IEEE Symposium on (pp. 77-81). IEEE

Dekker, R. (1996). Applications of maintenance optimization models: a review and analysis. Reliability Engineering \& System Safety, 51(3), 229-240.

Dehghanian, P., Fotuhi-Firuzabad, M., Bagheri-Shouraki, S., \& Razi Kazemi, A. A. (2012). Critical component identification in reliability centered asset management of power distribution systems via fuzzy AHP. Systems Journal, IEEE, 6(4), 593-602

Gaonkar, R. S. P., Verma, A. K., \& Srividya, A. (2008). Exploring fuzzy set concept in priority theory for maintenance strategy selection problem.International Journal of Applied Management 
and Technology, 6(3), 7.

Hunt, R. A., \& Xavier, F. B. (2003). The leading edge in strategic QFD.International Journal of Quality \& Reliability Management, 20(1), 56-73.

Karapetrovic, S., \& Rosenbloom, E. S. (1999). A quality control approach to consistency paradoxes in AHP. European Journal of Operational Research,119(3), 704-718.

Kathawala, Y., \& Motwani, J. (1994). Implementing quality function deployment: a systems approach. The TQM Magazine, 6(6), 31-37.

Kwong, C. K., \& Bai, H. (2002). A fuzzy AHP approach to the determination of importance weights of customer requirements in quality function deployment.Journal of intelligent manufacturing, 13(5), 367-377.

Lootsma, F. A. (1980). Saaty's priority theory and the nomination of a senior professor in operations research. European Journal of Operational Research,4(6), 380-388.

Lu, M. H., \& Kuei, C. H. (1995). Strategic marketing planning: a quality function deployment approach. International Journal of Quality \& Reliability Management, 12(6), 85-96.

McKone, K. E., Schroeder, R. G., \& Cua, K. O. (2001). The impact of total productive maintenance practices on manufacturing performance. Journal of operations management, 19(1), 39-58.

Mechefske, C. K., \& Wang, Z. (2003). Using fuzzy linguistics to select optimum maintenance and condition monitoring strategies. Mechanical Systems and Signal Processing, 17(2), 305-316.

Ni, G., Xie, M., Wang, W., Wang, J., \& Zhang, J. (2010, August). Research on the Risk Assessment Method of Vicarious Management Corporation Based on the Fuzzy AHP Method. In Information Science and Management Engineering (ISME), 2010 International Conference of (Vol. 2, pp. 531535). IEEE.

Pramod, V. R., Devadasan, S. R., \& Jagathy Raj, V. P. (2006). Customer voice adoption for maintenance quality improvement through MQFD and its receptivity analysis. International Journal of Management Practice, 2(2), 83-108.

Pramod, V. R., Devadasan, S. R., \& Jagathy Raj, V. P. (2007). Receptivity analysis of TPM among internal customers. International Journal of Technology, Policy and Management, 7(1), 75-88.

Pramod, V. R., Sampath, K., Devadasan, S. R., \& Jagathy Raj, V. P. (2007). Multicriteria decision making in maintenance quality function deployment through the analytical hierarchy process. International Journal of Industrial and Systems Engineering, 2(4), 454-478.

Pramod, V. R., Devadasan, S. R., Jagathy Raj, V. P., \& Murugesh, R. (2008). MQFD and its receptivity analysis in an Indian electronic switches manufacturing company. International Journal of Management Practice, 3(1), 82-95.

Ross, T. J. (2009). Fuzzy logic with engineering applications. John Wiley \& Sons.

Saaty, T. L. (1994). How to make a decision: the analytic hierarchy process.Interfaces, 24(6), 19-43.

Saaty, T. L. (2008). Decision making with the analytic hierarchy process.International journal of services sciences, 1(1), 83-98.

Sherwin, D. (2000). A review of overall models for maintenance management.Journal of Quality in Maintenance Engineering, 6(3), 138-164.

Thalia, S., Tuteja, A., \& Dutta, M. (2013, February). Comparative performance analysis of information system security using crisp and fuzzy AHP methods. In Advance Computing Conference (IACC), 2013 IEEE 3rd International (pp. 983-988). IEEE.

Triantaphyllou, E., \& Lin, C. T. (1996). Development and evaluation of five fuzzy multiattribute decision-making methods. international Journal of Approximate reasoning, 14(4), 281-310.

Verma, A. K., Srividya, A., \& Prabhu Gaonkar, R. S. (2007). Technical Note: Fuzzy Set Solutions for Optimal Maintenance Strategy Selection. Opsearch, 44(3), 261.

Wang, Y., \& Luan, X. (2012, August). The Green Supply Chain Evaluation and Construction of a Coal Chemical Enterprise in Hebei Province which Based on Fuzzy-AHP Method. In Industrial Control and Electronics Engineering (ICICEE), 2012 International Conference on (pp. 11461149). IEEE.

Yang,L., \& Zhang,Y. (2010). Applicationof Fuzzy-AHP Method in the Evaluation of Logistics Capability in E-Commerce Environment.InCommunications and Intelligence Information Security 
(ICCIIS), 2010 International Conference on (pp. 228-231). IEEE. 